\title{
Hemiballismus and choreoathetosis as a relapse in multiple sclerosis: A case report and review of literature
}

\author{
Ali Reza Nikseresht, Vahidreza Ostovan, Yadollah Asgari \\ Clinical Neurology Research Center, Department of Neurology, \\ Shiraz University of Medical Sciences, Shiraz, Iran
}

\begin{abstract}
Introduction. Multiple sclerosis (MS) is an autoimmune inflammatory demyelinating disease of the central nervous system (CNS). In contrast to tremor, which is frequently observed in MS patients, other movement disorders including parkinsonism, dystonia, chorea, ballism, myoclonus, and hemifacial spasm rarely occur.

Case report. The patient is a 34-year-old woman, a known case of MS manifested with choreiform movements in the tongue in conjunction with right upper and lower extremities choreoathetosis and hemiballismus. Magnetic resonance imaging (MRI) depicted a plaque in the contralateral subthalamic nucleus. The patient was treated with pulse methylprednisolone, antipsychotic and dopamine receptor blocker medications with complete recovery of her symptoms within one month.

Conclusions. Chorea or ballismus occur rarely as the presenting or relapsing features in MS patients. Therefore, clinicians should be familiar with this possibility and include high resolution neuroimaging and EEG in the diagnostic work-up of patients with MD to avoid misdiagnosis and inappropriate management.
\end{abstract}

Keywords: multiple sclerosis, hemiballismus, choreoathetosis, subthalamic nucleus, dyskinesias

\section{INTRODUCTION}

Multiple sclerosis (Ms) is an autoimmune indemyelinating disease of CNS which is diagnosed based on a set of clinical presentations and imaging

Tremor is the most common movement disorder in MS patients, and other movement disorders (MD) have been observed only in $1.6 \%$ of these patient [1]. Chorea comprises irregular, purposeless, rapid, brief, jerky, unsustain movements that can occur in each part of the body and move erratically from one part to another. The term "choreoathetosis" describes the combination of chorea and athetosis. The latter is a slow form of chorea characterized by writhing movements, spein the distal muscles of the extremities. Ballism refers to choreiform movements with wide amplitude that involves the proximal part of the limbs and most often affects only one side of the body which is called hemiballism [2]. To the best of our knowledge, a few case reports of choreoathetosis and hemiballismus have been reported in the literature so far in MS patients. Herein, we describe a rare patient with past medical history of MS presented with choreoathetosis and hemiballismus.

\section{CASE REPORT}

A 34-year-old woman was diagnosed with MS in 2010. The initial symptoms were bilateral blurred vision and lower extremities numbness and paresthesia. Laboratory studies including complete blood count (CBC), thyroid function tests (TFT), and vasculitis work-up were normal. Visual evoked potentials (VEP) were unobtainable in both eyes. Brain magnetic resonance imaging (MRI) demon- 
strated numerous high-intensity lesions which was consistent with diagnosis of MS according to 2010 McDonald criteria.The patient was treated with 5 grams of methylprednisolone followed by interferon beta- $1 \mathrm{~b}$. Thereafter, her symptoms subsided and she had no new neurological complaints. In February 2018 , she returned with three day history of dysarthria and persistent involuntary movements in the right side of her body, prominently in the right upper extremity which interfered with her daily activities. She had neither confusion nor amnesia in association with abnormal movements. Neurological exam revealed choreiform movements in the tongue as well as the right upper and lower extremities. Also, evidence of right-sided hemiballsimus was noted, particularly when attempting to maintain a posture predominantly in the upper limb. Furthermore, saccadic pursuit was noted on the gaze to the right side. Deep tendon

were brisk symmetrically and plantar were extensor bilaterally. Otherwise, the neurologic examination was unremarkable. Blood tests were normal and the electroencephalogram (EEG) demonstrated neither epileptiform discharges nor asymmetrical background. Brian MRI depicted multiple ent periventricular, subcortical and infratentorial high-intensity lesions in T2 and attenuated inversion recovery (FLAIR) sequences typical of demyelinating plaques. Also, in comparison to previous brain MRI, one new contrast enhancing high-intensity lesion was noted in the left subthalamic nucleus (Fig. 1A-C). Cervical MRI was normal. She was treated with1gram/day of intravenous(IV) methylprednisolone for consecutive days. Also, symptomatic treatment with tetrabenazine and risperidone was started for her. She im-
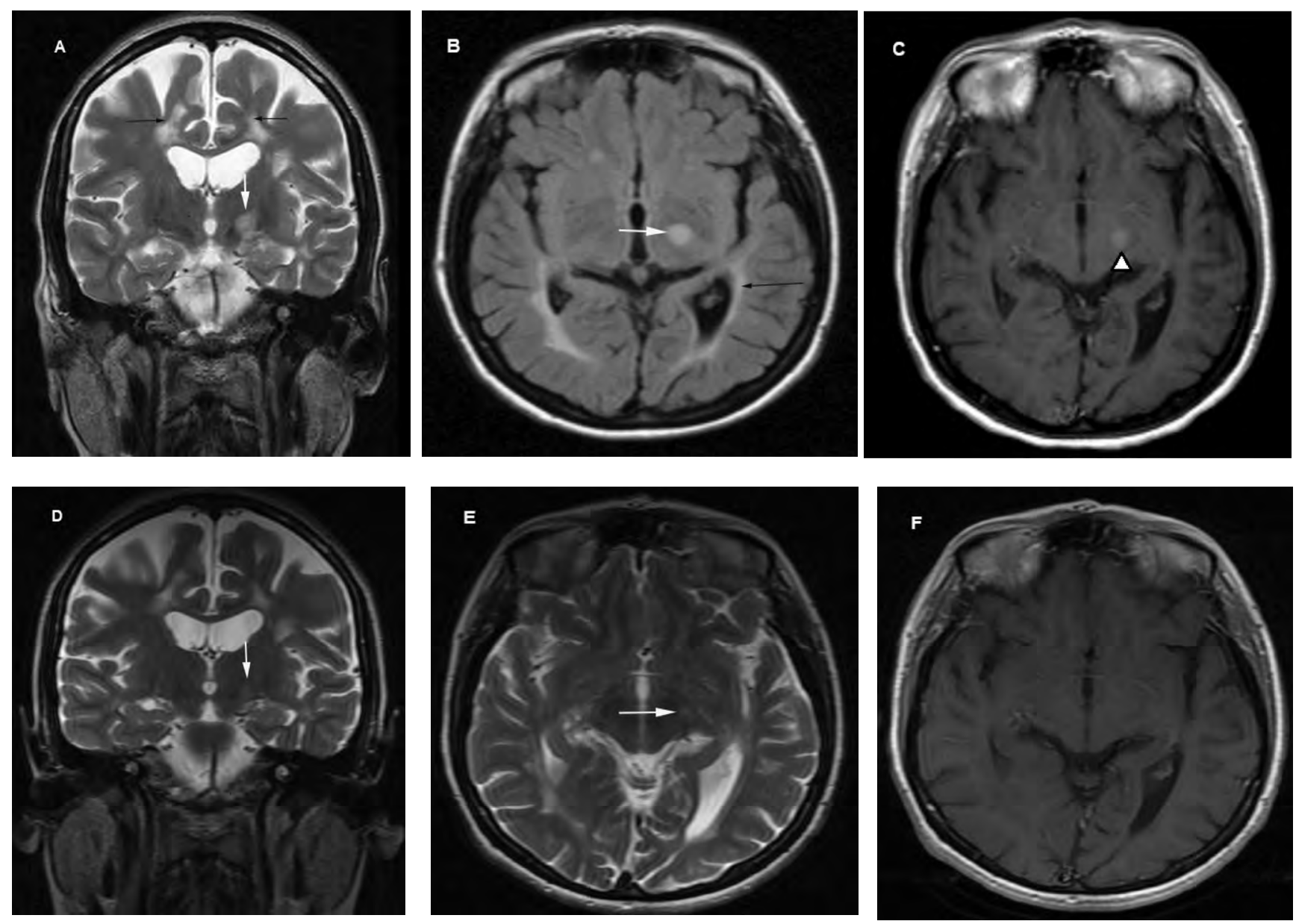

FIGURE 1A-F. Brain MRI

(A) Coronal T2-weighted and (B) axial FLAIR sequences show large hyper-intense lesion involving the left subthalamic nucleus(white arrow) and confluent periventricular plaques(black arrows). (C) Contrast enhanced axial T1-weighted image shows enhancement of left subthalamic nucleus lesion (white head arrow). (D) Coronal and (E) axial

T2-weighted sequences acquired two years later show significant resolution and shrinkage of the left subthalamic nucleus lesion (white arrow). (F) After 2 years of follow-up, contrast enhanced axial T1-weighted image shows complete resolution of the left subthalamic nucleus enhancement. 
proved completely over the next month; therefore, tetrabenazine and risperidone dosage was tapered gradually. The patient had no new attack over the next two years. Follow-up brain MRI showed neither new nor contrast enhancing lesion and shrinkage of the subthalamic plaque (Fig. 1D-F). Informed consent was obtained from the patient for her anonymous information to be published in this article.

\section{DISCUSSION}

Apart from tremor, other MD such as ballismus and chorea, rarely occur in MS patients [3]. The diagnosis of chorea and ballism is basically clinical and it is common to be misdiagnosed as epileptic seizures or psychogenic disorders, especially if not accompanied with other neurologic problem [4]. In our reported case, the possibility of epileptic seizures was ruled out with normal EEG during the attacks. The exact pathophysiology of chorea and ballismus in MS is still unknown and these abnormal movements can occur in some patients without obvious lesions in the basal ganglia [5]. Anatomically, choreoathetosis is due to an increase in the pallidal and thalamic activities caused by pathological abnormalities in the striatal neurons that are responsible for regulation of pallidal activities.
Moreover, it can also be due to the loss of regulating inputs from subthalamic nucleus [6], similar to our reported case. In addition to white matter, demyelinating plaque of MS can be seen in the deep gray matter nuclei including striatum, pallidum and thalamus with respect to the presence of myelinated in these areas [3]. The most prevalent anatomical site responsible for hemiballismus is contralateral subthalamic nucleus. Furthermore, lesions of subthalamic afferent and efferent striatum, globus pallidus, thalamus, and postcentral gyrus can promote hemiballismus [5]. Twelve cases of chorea or ballismus in association with MS have been reported so far in the literature. However, only seven of them had detailed clinical descriptions, time course of diagnosis of MS versus appearance of $\mathrm{MD}$, and precise location of demyelinating plaque (Table 1).

Among these documented cases, only patient reported by Pop et al. [7] had simultaneous chorea and ballismus with contralateral subthalamic nucleus lesion, similar to our presented case.

The response to treatment is another factor for determination of the relationship between MD and MS besides the neuroimaging [8,9]. When MD disappears spontaneously or with corticosteroid treatment, it implies the casual relationship between MD and MS, regardless of the presence or

TABLE 1. Reported cases of chorea and ballism in multiple sclerosis patients, MRI features and time course of $M D$ and $M S$

\begin{tabular}{|c|c|c|c|c|c|}
\hline Reference & Age(year) & Sex & Movement disorder & & $\begin{array}{l}\text { Onset of MD vs } \\
\text { diagnosis of MS } \\
\text { (year) }^{*}\end{array}$ \\
\hline $\begin{array}{l}\text { Riley and Lang } \\
\text { [5] }\end{array}$ & 25 & $F$ & Le & R. Subthalamic nuclei & +4 \\
\hline $\begin{array}{l}\text { Waubant et al. } \\
{[10]}\end{array}$ & 56 & $M$ & Le & R. Subthalamic nuclei & +4 \\
\hline de Seze et al.[11] & 16 & $M$ & $\begin{array}{l}\text { Le } \quad \text { xtremity } \\
\text { choreoathetosis }\end{array}$ & $\begin{array}{l}\text { R. Thalamus, Internal } \\
\text { pallidum, and posterior part } \\
\text { of the Internal capsule }\end{array}$ & 0 \\
\hline Pop et al. [7] & 20 & $\mathrm{~F}$ & $\begin{array}{l}\text { Right arm chorea and } \\
\text { ballism }\end{array}$ & L. Subthalamic nuclei & +2 \\
\hline Roos et al. [12] & 35 & $\mathrm{~F}$ & $\begin{array}{l}\text { Paroxysmal kinesigenic } \\
\text { choreoathetosis in le } \\
\text { and leg }\end{array}$ & $\begin{array}{l}\text { R. Globus pallidus and } \\
\text { काThalamus }\end{array}$ & +1 \\
\hline Minagar et al. [8] & 41 & $\mathrm{~F}$ & $\begin{array}{l}\text { Choreoathetoid in right } \\
\text { hand and leg }\end{array}$ & & +4 \\
\hline Chromik et al. [9] & 45 & $\mathrm{M}$ & Chorea in 4 limbs and trunk & & 0 \\
\hline Present case & 34 & $F$ & $\begin{array}{l}\text { Hemiballism \& } \\
\text { choreoathetosis }\end{array}$ & L. Subthalamic nuclei & +8 \\
\hline
\end{tabular}

VS, versus; MD, movement disorders; MS, sclerosis; $\left({ }^{*}\right)$ 0: diagnosis of MS and occurrence of MD were contemporary; + (yrs): MD onset a er diagnosis of MS; F, female; M, male; $R$, right; $L$, le magne es@nance imaging 
absence of the anatomical high signal lesions in MRI. However, the occurrence of MD in MS patients without obvious anatomical lesion in the responsible sites and lack of response to corticosteroid and therapy suggest that MD and MS are coincidental [9]. According to Potulska-Chromik et al. [9], in our presented case, MD is secondary to MS relapse as a result of both anatomical correlations and response to therapy. There is no comprehensive study into the treatment of choreoathetosis and hemiballismus in MS patients. In such patients, ballismus is cured spontaneously or after receiving steroid therapy; however, it may recur following the steroid withdrawal. Furthermore, MD may require to be treated temporarily with dopamine receptor blockers and tetrabenazine [10].

\section{CONCLUSIONS}

Chorea or ballismus occur rarely as the presenting or relapsing features in MS patients. Therefore, clinicians should be familiar with this possibility and include high resolution neuroimaging and EEG in the diagnostic work-up of patients with MD to avoid misdiagnosis and inappropriate management.

Conflict of interest: none declared Financial support: none declared

\section{REFERENCES}

1. Mehanna R, Jankovic J. Movement disorders in multiple sclerosis and other demyelinating diseases. J Neurol Sci. 2013;328(1-2):1-8

2. Jankovic J. Treatment of hyperkinetic movement disorders. Lancet Neurol. 2009;8(9):844-856.

3. Baguma M, Ossemann M. Paroxysmal kinesigenic dyskinesia as the presenting and only manifestation of multiple sclerosis after eighteen months of follow-up. J Mov Disord. 2017;10(2):96.

4. Alibhoy A, Wijemanne S, Gamage R. Paroxysmal kinesigenic dyskinesia. Ceylon Med J. 2006;51(1):36.

5. Riley D, Lang AE. Hemiballism in multiple sclerosis. Mov Disord. 1988;3(1):88-94.

6. Oakes PK, Srivatsal SR, Davis MY, et al. Movement disorders in multiple sclerosis. Phys Med Rehabil Clin N Am. 2013;24(4):639-651.

7. Pop R, Kipfer S. Paroxysmal kinesigenic dyskinesia-like phenotype in multiple sclerosis. Mult Scler. 2017;23(13):1795-1797.

8. Minagar A, Sheremata WA, Weiner WJ. Transient movement disorders and multiple sclerosis. Parkinsonism Relat Disord. 2002;9(2):111-113.
9. Potulska-Chromik A, Rudzinska M, Nojszewska M, et al. Clinical and neuroimaging correlation of movement disorders in multiple sclerosis: case series and review of the literature. Folia Neuropathol. 2014;52(1):92-100.

10. Waubant E, Simonetta-Moreau M, Clanet M, et al. Left arm monoballism as a relapse in multiple sclerosis. Mov Disord. 1997;12(6):1091-1092.

11. de Seze J, Stojkovic T, Destée M, et al. Paroxysmal kinesigenic choreoathetosis as a presenting symptom of multiple sclerosis. J Neurol. 2000;247(6):478.

12. Roos R, Wintzen A, Vielvoye $G$, et al. Paroxysmal kinesigenic choreoathetosis as presenting symptom of multiple sclerosis. J Neurol Neurosurg Psychiatry. 1991;54(7):657. 